\title{
CORRIGENDA
}

\section{Clinical impact of bone marrow morphology for the diagnosis of essential thrombocythemia: comparison between the BCSH and the WHO criteria}

H Gisslinger, G Jeryczynski, B Gisslinger, A Wölfler, S Burgstaller, V Buxhofer-Ausch, M Schalling, M-T Krauth, A-I Schiefer, C Kornauth, I Simonitsch-Klupp, C Beham-Schmid, L Müllauer and J Thiele

Leukemia (2017) 31, 774-775; doi:10.1038/leu.2016.291

Correction to: Leukemia (2016) 30, 1126-1132; doi:10.1038/ leu.2015.360

Following the publication of this Article, the authors noted that there were errors in Tables 2 and 4. The correct tables are provided below-amended values highlighted in bold and italicised.
Further the authors confirmed that the last sentence of the third to last paragraph of the methods section should be amended to read 'Antithrombotic therapy with low-dose aspirin was applied in 142 patients of the WHO-confirmed ET and 171 patients of the BCSH-defined ET cohort.'

The authors wish to apologize for any inconvenience caused.

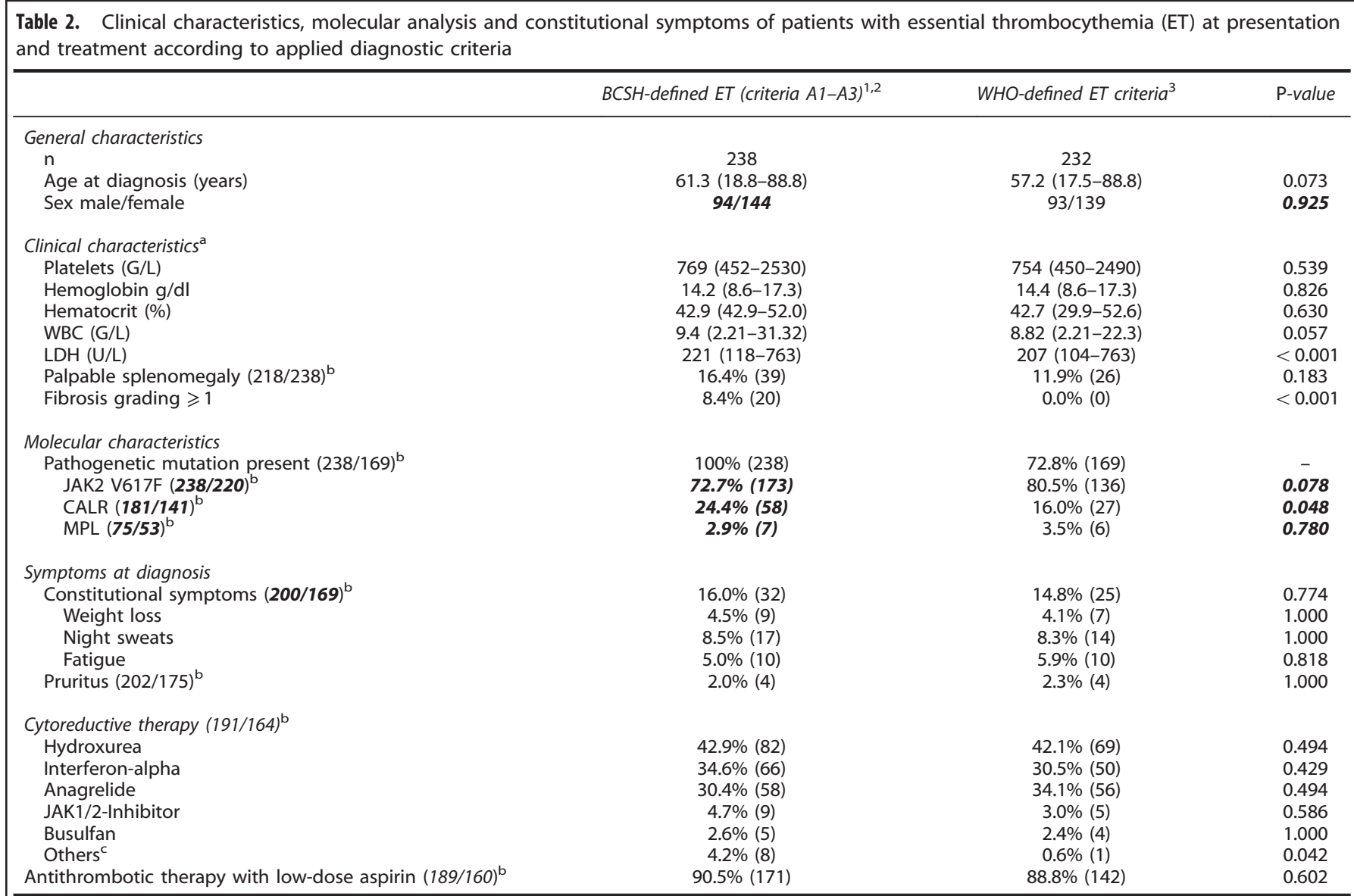

Abbreviations: WBC, white blood cell count; LDH, serum lactate dehydrogenase. ${ }^{a}$ Median, range. ${ }^{b}$ Number evaluable in each cohort. ${ }^{c}$ Pipobroman, P32 and other cytoreductive agents. 
Table 4. Clinical characteristics of patients with WHO-defined essential thrombocythemia (ET) compared with WHO-defined prefibrotic primary myelofibrosis (prePMF) at presentation as derived from the BCSH-confirmed ET cohort

\begin{tabular}{|c|c|c|c|}
\hline & WHO-defined $E T^{3}$ & WHO-defined prePMF ${ }^{3}$ & P-value \\
\hline \multicolumn{4}{|l|}{ General characteristics } \\
\hline $\mathrm{n}$ & 141 & 77 & \\
\hline Sex male/female & $58 / 83$ & $27 / 50$ & 0.486 \\
\hline \multicolumn{4}{|l|}{ Clinical characteristics $^{\mathrm{a}}$} \\
\hline Platelets (G/L) & $725(452-1836)$ & $840(457-2530)$ & 0.012 \\
\hline WBC $(\mathrm{G} / \mathrm{L})$ & $8.8(2.2-21.1)$ & $10.3(4.0-31.3)$ & 0.004 \\
\hline $\mathrm{LDH}(\mathrm{U} / \mathrm{L})$ & $209(110-763)$ & $270(136-598)$ & $<0.001$ \\
\hline Palpable splenomegaly $(141 / 77)^{\text {b }}$ & $9.9 \%(14)$ & $23.4 \%(18)$ & 0.009 \\
\hline Fibrosis grading $\geqslant 1$ & $0.0 \%(0)$ & $20.8 \%(16)$ & $<0.001$ \\
\hline \multicolumn{4}{|l|}{ Molecular characteristics } \\
\hline Pathogenetic mutation present $(141 / 77)^{\mathrm{b}}$ & $100 \%(141)$ & $100 \%(77)$ & - \\
\hline \multicolumn{4}{|l|}{ Symptoms at diagnosis } \\
\hline Constitutional symptoms $(111 / 71)^{b}$ & $15.8 \%(16)$ & $20.3 \%(10)$ & 1.000 \\
\hline Weight loss & $3.6 \%(4)$ & $7.0 \%(5)$ & 0.315 \\
\hline Night sweats & $8.1 \%(9)$ & $4.2 \%(3)$ & 0.372 \\
\hline Fatigue & $5.4 \%(6)$ & $5.6 \%(4)$ & 1.000 \\
\hline Pruritus $(111 / 71)^{\mathrm{b}}$ & $1.8 \%(2)$ & $1.4 \%(1)$ & 1.000 \\
\hline \multicolumn{4}{|l|}{ Cytoreductive therapy $(108 / 63)^{\mathrm{b}}$} \\
\hline Hydroxyurea & $45.4 \%(49)$ & $38.1 \%(24)$ & 0.423 \\
\hline Interferon-alpha & $31.5 \%(34)$ & $34.9 \%(22)$ & 0.736 \\
\hline Anagrelide & $33.3 \%(36)$ & $28.6 \%(18)$ & 0.610 \\
\hline JAK $1 / 2$-Inhibitor & $4.6 \%(5)$ & $6.3 \%(4)$ & 0.727 \\
\hline Busulfan & $1.9 \%(2)$ & $3.2 \%(2)$ & 0.626 \\
\hline Others ${ }^{\mathrm{c}}$ & $0.9 \%(1)$ & $6.3 \%(4)$ & 0.062 \\
\hline
\end{tabular}

\section{Long-term findings from COMFORT-II, a phase 3 study of ruxolitinib vs best available therapy for myelofibrosis}

CN Harrison, AM Vannucchi, J-J Kiladjian, HK Al-Ali, H Gisslinger, L Knoops, F Cervantes, MM Jones, K Sun, M McQuitty, V Stalbovskaya, P Gopalakrishna and T Barbui

Leukemia (2017) 31, 775; doi:10.1038/leu.2016.323

Correction to: Leukemia (2016) 30, 1701-1707; doi:10.1038/ leu.2016.148

Following the publication of this article the authors noted that the IPSS risk assignment was incorrectly listed in the second paragraph of the Results section. The correct proportions of patients with intermediate-2-risk or high-risk MF should be 40 and $60 \%$, respectively, not 60 and $40 \%$ as listed in the original.

The authors wish to apologize for any inconvenience caused. 